Original Research Paper

\title{
Lyapunov Function for a Dengue Transmission Model where two Species of Mosquitoes are Present: Global Stability
}

\author{
${ }^{1}$ Puntani Pongsumpun, ${ }^{2}$ Rattiya Sungchasit and ${ }^{3}$ I Ming Tang \\ ${ }^{I}$ Department of Mathematics, Faculty of Science, King Mongkut's Institute of Technology Ladkrabang, \\ Chalongkrung Road, Ladkrabang, Bangkok 10520, Thailand \\ ${ }^{2}$ Department of Mathematics, Faculty of Science and Technology, \\ Phuket Rajabhat University Muang District, Phuket, 83000, Thailand \\ ${ }^{3}$ Computational and Applied Science for Smart Innovation Cluster (CLASSIC), Faculty of Science, \\ King Mongkut's University of Technology Thonburi, Bangkok 10140, Thailand
}

Article history

Received: 08-05-2017

Revised: 19-06-2017

Accepted: 24-10-2017

Corresponding Author:

P. Pongsumpun,

Department of Mathematics,

Faculty of Science, King

Mongkut's Institute of

Technology Ladkrabang,

Chalongkrung Road,

Ladkrabang, Bangkok 10520,

Thailand

E-mail: kppuntan@kmitl.ac.th

\begin{abstract}
In this study, we are interested in the newly observed endemic due to the ZIKA virus. In the absence of a full knowledge of the dynamics of the infection due to this virus, we consider a model of the closely related dengue virus when there are two species of mosquitoes, A. aegypti and $A$. albopictus mosquitoes who are both capable of transmitting the two viruses. We use a Susceptible-Infected-Recovered (SIR) model to describe the infection of the human populations. We obtain the basic reproduction ratio $\left(R_{0}\right)$ and show that if $R_{0}$ is less than 1 , the disease free equilibrium state is global asymptotically stable. If $R_{0}$ is greater than 1 , we use the Lyapunov function approach to find the conditions for the unique dengue endemic equilibrium state to be globally stable. We then point out the insights into the global stability of the ZIKA epidemic that can be gained by looking at the global stability of a model for the dengue infection in the presence of two species of mosquitoes that can transmit the disease.
\end{abstract}

Keywords: Asymptotically Stable, Dengue Disease, Equilibrium State, Global Stability, Species, Lyapunov Function

\section{Introduction}

The big scare in the world public health community right now (Janurary 2016) is the ZIKA virus WHO (2016). This virus was discovered in the Zika forest in Uganda, Africa. In the 2007 outbreak in the Federated States of Micronesia, 5000 ZIKA infections were reported in a population of 6700 Peterson et al. (2016) Since the virus at the time was thought to cause only a mild febrile fever Simpson (1964), not much attention was paid to the virus. However, in the recent outbreak in Brazil, some of the babies born to pregnant female patients developed microcephaly (a disease which causes the heads of newly born babies to be undersize and the brain to be malformed Mliakar (2016). The possible connection between the ZIKA virus and microcephaly was the reason for World Health Organization (WHO) on Feb. 1, 2016 to declare the possible ZIKA pandemic to be a global health emergency (Science News, 2016). On April 13, 2016, CDC (Center for Disease Control (USA)) announced that the ZIKA virus met the conditions for causality of microcephaly by the ZIKA virus Rasmussen et al. (2016). This new complication arising from this disease led the President (New York Times, 2016) of the USA to ask the US Congress for 1.8 billion dollar to combat this disease and scientists to promise that a vaccine against this disease could be obtained in eighteen months.

The virus belongs to the family Flavivindae genius Flavivirus. Another member of this family and genius is the dengue virus. The vectors of both of these viruses are mosquitoes of the Aedes family, the A. aegypti mosquitoes in urban areas Koenraadt et al. (2007; Chadee, 2004) and the A. albopictus mosquitoes in rural areas Hawley (1988; Mori and Wada, 1972). The (normal) symptoms caused by these two viruses are similar, i.e., mild fever, skin rashes, muscle and joint pains and headaches. In this paper we propose to gain some insights in the spread of the ZIKA virus in Brazil when the presence of both the $A$. aegypti and $A$. albopictus mosquitoes are taken into account Serpa et al. (2013). The reason for taking into account the presence of two species is due to difference in the effects of the different environments in the different parts of Florida, USA on the two species caused the pattern of the 
infections to be different in the different parts of Florida Costanzo et al. (2005; Juliano et al., 2002).

Since a full understanding of the ZIKA epidemic (how it is spread) is not known, we propose to look at the global stability of the solutions of a transmission model of the related disease, dengue fever Keeling and Rohani (2008; Diekmann and Heesterbeek, 2000) in which there are two species of mosquitoes transmitting the virus. Returning to the point raised in the first sentence, in order to model the transmission of a disease, it is necessary to know all the important factors that affect the transmission. As pointed out by Fauci and Morens (2016), the Zika virus is the last of the four recent arthropod-borne viral disease (Zika, dengue, West Nile and Chikungunya) in the Western Hemisphere in past twenty years. The Zika, Dengue and West Nile viruses are flavivi-ruses. The first two are also transmitted by same aedes mosquitoes. Fauci and Morens suggested that one uses common platforms for quick flaviviruses development, i.e., through the expression of immunogenic antigens of the newly emerging virus. For development of the Zika vaccine, this brings up the problem of Antibody-Dependent Enhancement (ADE) since the Zika infections are occurring in areas where the dengue epidemic is also occurring Pierson and Graham (2016). ADE caused by different serotype of the flavivirus causes the viremia and severity of the disease to increase Vaughn et al. (2000). It has been shown Priyamvada (2016) that the human antibodies due to dengue fever are highly cross reactive to the ZIKA virus. A in vitro study Bardina et al. (2017) found that enhancement of ZIKA pathogenesis infections by the preexisting antiflavivirus The ADE in this case was mediated through the IgG engagement of the $F c_{y}$ receptors. Another study Littaua et al. (1990) found that the ADE leading to dengue hemorrhagic fever was the IgG to Fc receptor II. However, a newer study McCracken et al. (2017) using rhesus macaques did indicate that prior infection with a heterologous flaviviruses neither increase or decrease the ZIKA titers in these non human primates, Further investigations are needed to determine whether ADE should be included in a mathematic model for the transmission of the ZIKA disease. If it is, then the susceptible class must be divided into two classes, susceptible who have not been infected with the dengue virus and those who have not. This may present some difficulties since the majority of dengue and ZIKA infected patients are asymptotic cases, i.e., they do not exhibit any clinical symptoms.

Also evidence has been building that sexual transmission of the ZIKA virus is possible Musso et al. (2015; D’Ortenzio et al., 2016). Sexual transmission by women is possible since the ZIKA virus has been found in the vaginal fluids Turmel et al. (2016; Davidson et al., 2016). So far, only a few cases of sexual transmission have been reported in the West. There have been no reports of a secondary ZIKA infection resulting from STD ZIKA patient and so it is too early to include this into a disease transmission model.

The present authors have already established the conditions for local asymptotic stability of a dengue model in which two species of mosquitoes are present Sungchasit et al. (2013). In that paper, the authors showed that when there were seasonal variations in the rates at which of the dengue infections are transmitted from the two species to the human, limit cycle was seen $\mathrm{n}$ the trajectories of the two mosquitoes populations and that the endemic equilibrium state was locally asymptotically stable when the basic reproduction number is greater than one. We use the same parameter values used in that paper which lead to the fraction of $A$ aegypti mosquitoes oscillating between 0.261 and 0.04587 and the fraction of $A$. albopictus oscillating between 0.010246 and 00002 . In this paper, we wish to establish the global stability of this model using the Lyapunov approach. The aim is to gain insights into the stability of an illness caused by the related ZIKA virus whose nature or means of transmission are not completely known.

Li and Muldowney (1995) have studied the global stability of the solutions of the SEIR (susceptibleexposed-infected-recovered) model commonly used in epidemiological modeling. They showed that the endemic equilibrium which is locally asymptotically stable when the contact number (defined in their paper) $\sigma \leq 1$ and becomes globally asymptotically stable when $\sigma>1$. Beretta and Capasso (1986) found the conditions needed for the endemic equilibrium of a SIR model with constant population size to be globally stable. At the present stage of knowledge of the ZIKA epidemic, it is not known if there is an exposed stage in the humans so we will use a simpler model to describe the dynamics of this disease in humans, the SIR model. We will be looking at a similar model except that there will be two species of mosquitoes present. In this paper, we use a generalized Lyapunov function to analysize the global dynamic of the eigth - dimensional model of dengue disease of the human population and different Aedes mosquitoes (Aedes aegypti and Aedes albopictus), where an infected human can be one who was infected by a bite of an A. aegypti mosquitoes or by an A. albopictus mosquitoes. We will prove the global stability of the equilibrium states using Lyapunov functions LaSalle and Lefschetz (1961). Discussion and conclusion are given in the last section. 


\section{Model Formulation and Stability Analysis}

We use the Susceptible-Infected-Recovered (SIR) for human and Susceptible-Infected (SI) for both species of the Aedes mosquitoes. In fact, there has been no report of which species of the mosquitoes is involved in the transmission of the ZIKA virus. We consider the presence of the two (the A. aegypti and A. albopictus mosquitoes) since both are present in Brazil. One of the reasons for mentioning only the first specie is that the alarm is centered in the urban areas of Brazil. The initial outbreak was in the city Recife in northeastern Brazil. Evidence has been accumulating that $A$. albopictus has adapted itself so that it can thrive in urban environments Benedict et al. (2007). The great fear is that it will spread to Rio de Janeiro, the site of the 2016 Olympics where many people from the rest of the world will come. Cancelling the Olympics is out of the question. There are studies which show that both species of the mosquitoes are present in parts of Brazil (Costanzo et al., 2005) and if we are to look at the transmission of the diseases in Brazil, we should take into account the presence of both species.

The dengue virus Gubler (1998) is spread by the bite of adult female Aedes mosquitoes only, since the female needs the blood to complete the ovigenesis cycle. Only in the viraemic period which ranges from 2 to 12 days with an average of 4-5 days, is an infected person capable of transmitting the virus. During this period, a dengue-infected person is capable of transmitting the dengue virus to the two species ( $A$. aegypti and $A$. albopictus) of vectors when they are bitten by the mosquitoes Guga-Sapir and Schimmer (2005). During the blood meal, the females ingest the dengue virus from an infectious human where the virus goes into the gut of the mosquitoes. The dengue virus then undergoes an extrinsic incubation period (of approximately 8-12 days in the Aedes mosquitoes) before the mosquitoes become infectious and can transmit the virus to the humans. The presence of the incubation period causes a time which we ignore since it has not been established that this occurs with the ZIKA virus. If the temperature is low enough, the incubationperiod can be longer then the life time of the mosquitoes, in which case no transmission of the virus will occur.

\section{Parameters of Model}

Let $N_{T}, N_{v a}$ and $N_{v b}$ represent the total number of human. In this paper, the total number of population is to be constant for each category. The human population of sizes $N_{T}$ consists of susceptible humans (S), infected humans due to a bite of an A. aegypti $\left(I_{a}\right)$ mosquito or of an $A$. albopictus $\left(I_{b}\right)$ mosquito and Recovered Human $(R)$, i.e., $N_{T}=S+I_{a}+I_{b}+R$. For vector population of sizes $N_{v a}$, is total number of an adult $A$. aegypti mosquito which consist of the susceptible $\left(S_{v a}\right)$ and infectious mosquitoes $\left(I_{v a}\right)$, i.e., $N_{v b}=S_{v b}+I_{v b} . . N_{v b}$ is the total number of $A$. albopictus mosquitoes, i.e., $N_{v b}=S_{v b}+I_{v b}$ and $S_{v b}$ is susceptible mosquito, $I_{v b}$ is infectious mosquito. Note that $\mu_{h}$ and $\mu_{d}$ are the average constant natural death rate of human population and death rate of human population due to the disease, $\mu_{v a}$ and $\mu_{v b}$ are the average constant death rate in $A$. aegypti and $A$. albopictus mosquitoes, respectively. As we have mentioned earlier, the seasonal variation is simulated by introducing sinusoidal dependence into the rates of transmission of the virus to the humans and not into the death rates as is done by most researchers. The reason for this was given earlier.

The other parameters are defined as follows: $\kappa$ is the birth rate of human population, $N_{h}$ is the total human population at time $t . \omega_{a}$ and $\omega_{b}$ are the biting rates of Aedes aegypti population and Aedes albopictus population, $\lambda_{a}$ and $\lambda_{b}$ are the measure of influence on the transmission process from human population to Aedes aegypti and Aedes albopictus, $\alpha_{h a}$ and $\alpha_{h b}$ are the recovery rate of human population who be infected by an $A$. aegypti and $A$. albopictus mosquitoes. It is well known that a person infected with one serotype of the Dengue virus cannot be re infected by the same serotype but can be infected by a different serotype which may result in death causing illness. For vector population, it is assumed a constant recruitment rate $Q_{a}$ and $Q_{b}$ of $A$. aegypti and A. albopictus, $\lambda_{v a}$ and $\lambda_{v b}$ are the measure of influence on the transmission process from $A$. aegypti and $A$. albopictus, respectively to human population, $\beta_{v a}$ and $\beta_{v b}$ are the transmission probability of dengue disease from vector populations (A. aegypti and $A$. albopictus) to human population, as well as the number of infectious and susceptible of each species. In reality, the rate at which the adult mosquitoes are recruited depends on how many eggs are laid, on whether the eggs hatch and how many of the larvae survive the metamorphosis process to become adults. For our simple model, we ignore all of this and insert the sinusoidal dependence into the rates of transmission since these depend on the number of bites the mosquitoes must make to obtain the necessary amount of blood needed for the ovigenesis cycle. Our model equations are shown in the next section.

\section{Equations of Model}

In this paper, we study the transmission of dengue disease in a human population where there are two species of mosquitoes, A. aegypti and A. albopictus mosquitoes. We assume that there is only one type of dengue virus present, even though there are four serotypes; DEN1, DEN2, DEN3 and DEN4. Infection by one type confers life-long immunity to further infection by that type. 
There is no immunity to infection by the others. However, infection by a different serotype opens up the possibility an allergic reaction to the antibodies of the first type, resulting in a more severe form of the disease, dengue hemorrhagic fever. Figure 1 the monthly incidence of dengue fever in Thailand in the period 2003 to $2011 \mathrm{MOPH}$ (2011). The figure does not show a systematic yearly increase of the disease. The incidences can be larger or smaller year to year. They all show a sinusoidal behavior. We have therefore included sinusoidal dependence in the rates of transmission of the virus to the humans from an infectious mosquito of either species and of the transmission of the virus to either specie of mosquito from an infected human. The rates of transmissions between a human and mosquito usually contains factor giving the number of contacts between the two. If there is no contact, the rates of transmission would be zero. Other studies Fisman (2007; Greenfell et al., 1995) have introduced a sinusoidal dependence in the death rates, reasoning that when the weather gets cold enough, the mosquitoes will die. Our choice of where to introduce the sinusoidal dependence is based on the fact that the Ades mosquitoes breeding habits are different from the other mosquito genius. The females of the Ades mosquitoes lay their eggs above the water line of the breeding pond. The eggs will hatch only after they are inundated with water from a new rain fall. If there is no new rain fall, there will be more mosquitoes unless humans replenish the water by artificially spraying the area.

The flow chart for the transmission of the dengue virus when there are two species of mosquitoes present is shown in Fig. 2.

\section{The Mathematical Representation of the Flow Chart}

The mathematical representation of the model is given by the following system of ordinary differential equations:

$$
\begin{gathered}
\frac{d S}{d t}=\kappa N_{h}-\omega_{a}\left(1+\lambda_{a} \sin \eta t\right) I_{v a} S-\mu_{d} S-\mu_{h} S-\omega_{b} \\
\left(1+\lambda_{b} \sin \eta t\right) I_{v b} S
\end{gathered}
$$

$\frac{d I_{a}}{d t}=\omega_{a}\left(1+\lambda_{a} \sin \eta t\right) I_{v a} S-\mu_{d} I_{a}-\mu_{h} I_{a}-\alpha_{h a} I_{a}$

$\frac{d I_{b}}{d t}=\omega_{b}\left(1+\lambda_{b} \sin \eta t\right) I_{v b} S-\mu_{d} I_{b}-\mu_{h} I_{b}-\alpha_{h b} I_{b}$

$\frac{d R}{d t}=-\mu_{d} R-\mu_{h} R+\alpha_{h a} I_{a}+\alpha_{h b} I_{b}$

$\frac{d S_{v a}}{d t}=Q_{a}-\beta_{v a}\left(1+\lambda_{v a} \sin \eta t\right) S_{v a} I_{a}-\mu_{v a} S_{v a}$

$\frac{d I_{v a}}{d t}=\beta_{v a}\left(1+\lambda_{v a} \sin \eta t\right) S_{v a} I_{a}-\mu_{v a} I_{v a}$

$\frac{d S_{v b}}{d t}=Q_{b}-\beta_{v b}\left(1+\lambda_{v b} \sin \eta t\right) S_{v b} I_{b}-\mu_{v b} S_{v b}$

$\frac{d I_{v b}}{d t}=\beta_{v b}\left(1+\lambda_{v b} \sin \eta t\right) S_{v b} I_{b}-\mu_{v b} I_{v b}$

All parameters in our model are non- negative. We will now show that the solutions to the equations given by $(1)-(8)$, in the non-negative octant $R_{+}^{8}$ are positive invariant (where $\mathrm{R}_{+}^{8}$ denotes the non-negative region). With respect to system $(1)-(8)$, we have the following results.

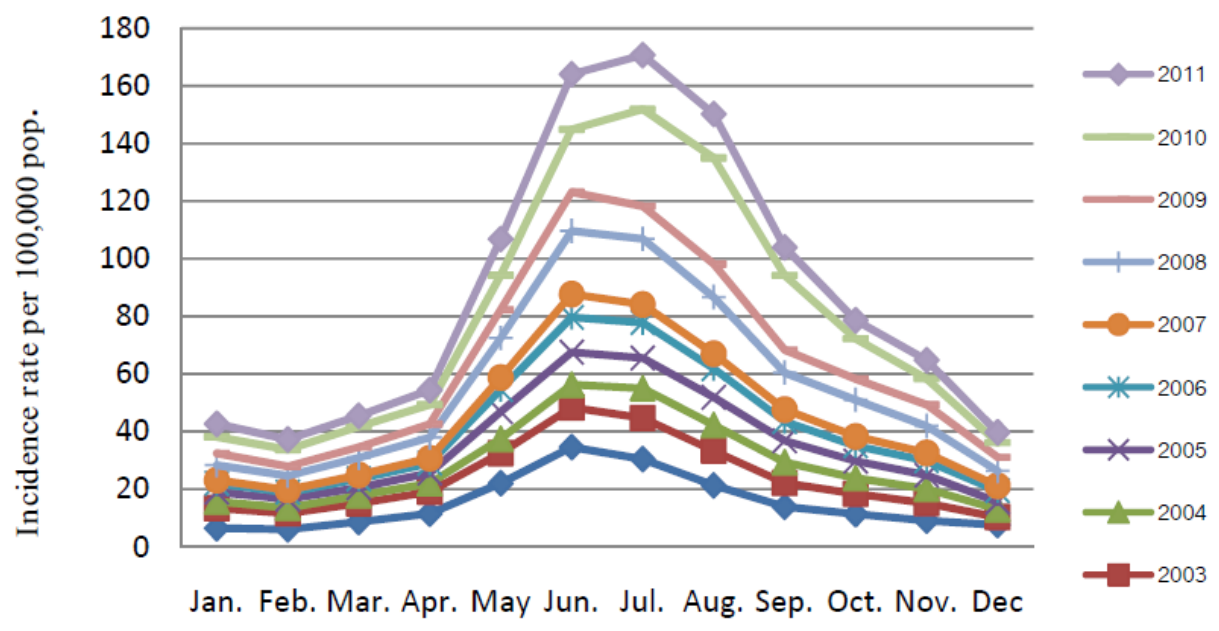

Fig. 1. Reported cases of Dengue disease per 100,000 population in Thailand during 2003 and 2011 (month - by - month) [Division of Epidemiology, 2002-2011] 


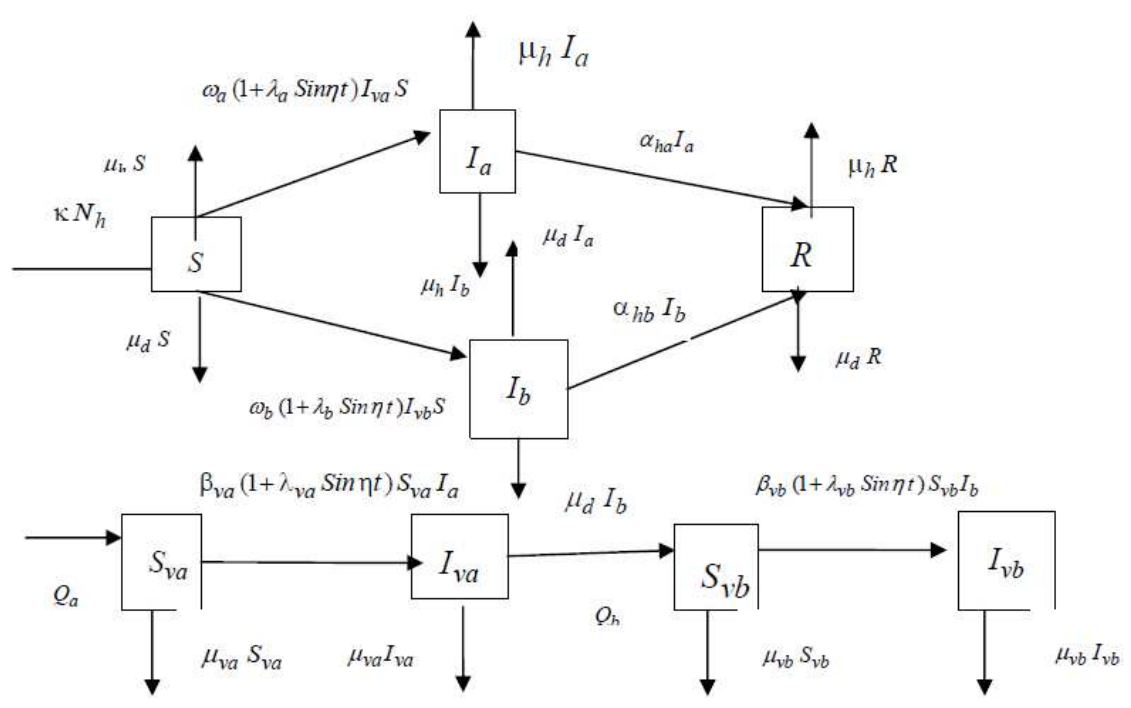

Fig. 2. SIR model

\section{Proposition 1}

Let

$\left.(S(t)), I_{a}(t), I_{b}(t), R(t), S_{v a}(t), I_{v a}(t), S_{v b}(t), I_{v b}(t)\right)$ be the solution of (1) - (8) with the initial condition $\left(S(0), I_{a}(0), I_{b}(0), R(0), S_{v a}(0), I_{v b}(0), S_{v b}(0), I_{v b}(0)\right) \quad$ and the compact set:

$$
\Omega_{a}=\left\{\begin{array}{l}
\left(S, I_{a}, I_{b}, R, S_{v a}, I_{v a}, S_{v b}, I_{v b}\right) \in R_{+}^{8}, W_{1} \leq N_{T} \\
=\frac{\kappa N_{h}}{\mu_{d}+\mu_{h}}, W_{2} \leq N_{v a}=\frac{Q_{a}}{\mu_{v a}}, W_{3} \leq N_{v b}=\frac{Q_{b}}{\mu_{v b}}
\end{array}\right\}
$$

Then, under the flow described by (1) $-(8), \Omega_{a}$ is a positively invariant set that attracts all solutions in $\mathrm{R}_{+}^{8}$.

\section{Proof}

We choose the Lyapunov function:

$$
\begin{aligned}
W(t) & =\left(W_{1}(t), W_{2}(t), W_{3}(t)\right) \\
& =\left(S+I_{a}+I_{b}+R, S_{v a}+I_{v a}, S_{v b}+I_{v b}\right)
\end{aligned}
$$

to be positive definite on $\mathrm{R}_{+}^{8}$ and we have:

$$
\begin{aligned}
\frac{d W}{d t} & =\left[\frac{d W_{1}}{d t}, \frac{d W_{2}}{d t}, \frac{d W_{3}}{d t}\right] \\
& =\left[\frac{d S}{d t}+\frac{d I_{a}}{d t}+\frac{d I_{b}}{d t}+\frac{d R}{d t}, \frac{d S_{v a}}{d t}+\frac{d I_{v a}}{d t}, \frac{d S_{v b}}{d t}+\frac{d I_{v b}}{d t}\right] \\
& =\left(\begin{array}{l}
\kappa N_{h}-\left(\mu_{d}+\mu_{h}\right)\left(S+I_{a}+I_{b}+R\right), Q_{a}-\mu_{v a}\left(S_{v a}+I_{v a}\right), \\
Q_{b}-\mu_{v b}\left(S_{v b}+I_{v b}\right)
\end{array}\right) \\
& =\left(\kappa N_{h}-\left(\mu_{d}+\mu_{h}\right) N_{T}, Q_{a}-\mu_{v a} N_{v a}, Q_{b}-\mu_{v b} N_{v b}\right)
\end{aligned}
$$

We used the fact that $N_{T}=\frac{\kappa N_{h}}{\mu_{d}+\mu_{h}}, N_{v a}=\frac{Q_{a}}{\mu_{v a}}$ and $N_{v b} \frac{Q_{b}}{\mu v_{b}}$.

With this in mind, it is not difficult to show that:

$$
\begin{aligned}
& \frac{d W_{1}}{d t}=\kappa N_{h}-\left(\mu_{d}+\mu_{h}\right) W_{1} \leq 0, \quad \text { for } W_{1} \geq \frac{\kappa N_{h}}{\mu_{d}+\mu_{h}} \\
& \frac{d W_{2}}{d t}=Q_{a}-\mu_{v a} W_{2} \leq 0, \quad \text { for } \quad W_{2} \geq \frac{Q_{a}}{\mu_{v a}}
\end{aligned}
$$

$\frac{d W_{3}}{d t}=Q_{b}-\mu_{v b} W_{3} \leq 0, \quad$ for $\quad W_{3} \leq \frac{Q_{b}}{\mu_{v b}}$

From the above equations (9) - (11) one has that $\frac{W d}{d t} \leq 0$ which implies that $\Omega_{a}$ is a positively invariant set. In other words, by solving (9) - (11), we obtain:

$$
\begin{aligned}
& 0 \leq\left(W_{1}(t), W_{2}(t), W_{3}(t)\right) \\
& \leq\left(\begin{array}{l}
\left(\kappa N_{h} /\left(\mu_{d}+\mu_{h}\right)\right) \\
+W_{1}(0) e^{-\left(\mu_{d}+\mu_{h}\right) t},\left(Q_{a} / \mu_{v a}\right)+ \\
W_{2}(0) e^{-\mu_{v a} t}, \quad\left(Q_{b} / \mu_{v b}\right)+W(0) e^{-\mu_{v b} t}
\end{array}\right),
\end{aligned}
$$

where, $W_{1}(0), W_{2}(0)$ and $W_{3}(0)$ are respectively, the initial conditions of $W_{1}(t), W_{2}(t)$ and $W_{3}(t)$. Thus, as $t \rightarrow \infty, 0 \leq\left(W_{1}(t), W_{2}(t), W_{3}(t)\right) \leq\left(\kappa N_{h} / \mu_{d}+\mu_{h}, Q_{a} / \mu_{v a}\right.$, $\left.Q_{b} / \mu_{v b}\right)=\left(N_{T}, N_{v a}, N_{v b}\right)$ and one can conclude that $\Omega_{a}$ is an attractive set. 


\section{Equilibrium Points}

From equations (1) - (8), we set the right hand side of all equations to zero. We obtain two equilibrium points:

- (I) If $R_{0} \leq 1$, the only equilibrium is the disease free equilibrium point:

$$
\begin{aligned}
& J_{1}\left(S, I_{a}, I_{b}, R, S_{v a}, I_{v a}, S_{v b} I_{v b}\right) \\
& \quad=J_{1}=\left(\frac{\kappa N_{h}}{\mu_{d}+\mu_{h}}, 0,0,0, \frac{Q_{a}}{\mu_{v a}}, I_{v a}, \frac{Q_{b}}{\mu_{v b}}, 0\right) \in \Omega_{a} .
\end{aligned}
$$

- (II) If $R_{0}>1$, there is the endemic equilibrium point:

$$
J_{2}\left(S^{*}, I_{a}^{*}, I_{b}^{*}, R^{*}, S_{v a}^{*}, I_{v a}^{*}, S_{v b}^{*}, I_{v b}^{*}\right) \in \Omega_{a}
$$

where, $S^{*}, I_{a}^{*}, I_{b}^{*}, R^{*}, S_{v a}^{*}, I_{v a}^{*}, S_{v b}^{*}, I_{v b}^{*}>0$ satisfy:

$$
S^{*}=\frac{\left(\mu_{v a}\left(I_{a \gamma A A}^{*}+\mu_{v a}\right) \mu_{v b}\left(N_{h \gamma B B} \kappa+\left(\alpha_{h b}+\mu_{d}+\mu_{h}\right) \mu_{v b}\right)\right)}{\left(\begin{array}{l}
\gamma_{B B} \mu_{v a}^{2}\left(\gamma_{H B} Q_{b}+\left(\mu_{d}+\mu_{h}\right) \mu_{v b}\right)+I_{a \gamma A \gamma \gamma B B}^{*} \\
\left(\gamma_{H B} Q_{b} \mu_{v b}+\gamma_{H A} Q_{b} \mu_{v b}+\left(\mu_{d}+\mu_{h}\right) \mu_{v a} \mu_{v b}\right)
\end{array}\right)}
$$

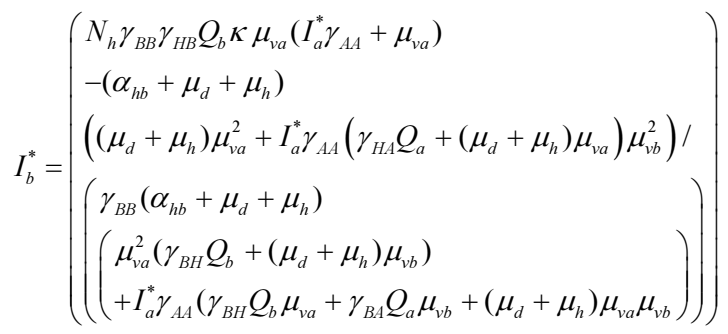

$$
R^{*}=\left\{\begin{array}{l}
\alpha_{h a}\left(\begin{array}{l}
-\gamma_{B B} \gamma_{H B} Q_{b}\left(\alpha_{h a}+\mu_{d}+\mu_{h}\right) \mu_{v a}-\gamma_{B B} \\
\left(N_{h} \gamma_{A A} \gamma_{H A} Q_{a} \kappa+\left(\mu_{d}+\mu_{h}\right)\left(\alpha_{h b}+\mu_{d}+\mu_{h}\right) \mu_{v b}^{2}\right) \\
\left.\mu_{v b}+\gamma_{A A} \gamma_{H A} Q_{a}\left(\alpha_{h b}+\mu_{d}+\mu_{h}\right) \mu_{v b}^{2}\right)
\end{array}\right) \\
\left(\begin{array}{l}
\gamma_{A A}\left(\alpha_{h a}+\mu_{d}+\mu_{h}\right)\left(\begin{array}{l}
\gamma_{H B} Q_{b} \mu_{v a}+\gamma_{H A} Q_{a} \mu_{v b}+ \\
\left(\mu_{d}+\mu_{h}\right) \mu_{v a} \mu_{v b}
\end{array}\right)+ \\
\left(\begin{array}{l}
N_{h b}\left(\begin{array}{l}
N_{h} \gamma_{B B} \gamma_{H B} Q_{b} \kappa \mu_{v a}\left(I_{a}^{*} \gamma_{A A}+\mu_{v a}\right)-\left(\alpha_{h b}+\mu_{d}+\mu_{h}\right) \\
\left(\left(\mu_{d}+\mu_{h}\right) \mu_{v a}^{2}+I_{a}^{*} \gamma_{A A}\left(\gamma_{H A} Q_{a}+\left(\mu_{d}+\mu_{h}\right) \mu_{v a}\right)\right.
\end{array}\right) \mu_{v b}^{2}
\end{array}\right) \\
/\left(\begin{array}{l}
\left(\alpha_{h b}+\mu_{d}+\mu_{h}\right)\left(\mu_{v a}^{2}\left(\gamma_{H B} Q_{b}+\left(\mu_{d}+\mu_{h}\right) \mu_{v b}\right)+\right. \\
I_{a}^{*} \gamma_{A A}\left(\gamma_{H B} Q_{b} \mu_{v a}+\gamma_{H A} Q_{a} \mu_{v b}\right)+\left(\mu_{d}+\mu_{h}\right) \mu_{v a} \mu_{v b}
\end{array}\right)
\end{array}\right) \\
/\left\{\begin{array}{l}
\left.\gamma_{B B}\left(\mu_{d}+\mu_{h}\right)\right\}
\end{array}\right.
\end{array}\right.
$$

$$
S_{v a}^{*}=\frac{Q_{a}}{I_{a}^{*} \gamma_{A A}+\mu_{v a}}
$$

$$
I_{v a}^{*}=\frac{I_{a}^{*} \gamma_{A A} Q_{a}}{I_{a}^{*} \gamma_{A A} \mu_{v a}+\mu_{v a}^{2}}
$$

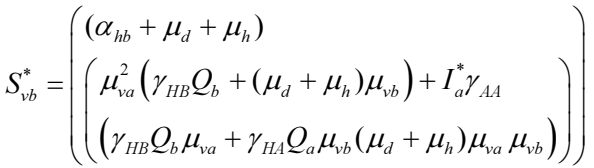

$$
\begin{aligned}
& /\left(\gamma_{H B} \mu_{v a}\left(I_{a}^{*} \gamma_{A A}+\mu_{v a}\right)\left(N_{h} \gamma_{B B} K+\left(\alpha_{h b}+\mu_{d}+\mu_{h}\right) \mu_{v b}\right)\right) \\
& I_{v b}^{*}=\left(\begin{array}{l}
N_{h} \gamma_{B B} \gamma_{H B} Q_{b} \kappa \mu_{v a}\left(I_{a}^{*} \gamma_{A A}+\mu_{v a}\right) \\
-\left(\alpha_{h b}+\mu_{d}+\mu_{h}\right)\left(\begin{array}{l}
\left(\mu_{d}+\mu_{h}\right) \mu_{v a}^{2} \\
+I_{a}^{*} \gamma_{A A}\left(\gamma_{H A} Q_{a}+\left(\mu_{d}+\mu_{h}\right) \mu_{v a}\right)
\end{array}\right) \mu_{v b}^{2}
\end{array}\right) \\
& /\left(\gamma_{H B} \mu_{v a}\left(I_{a}^{*} \gamma_{A A}+\mu_{v a}\right) \mu_{v b}\left(N_{h} \gamma_{B B} K+\left(\alpha_{h b}+\mu_{d}+\mu_{h}\right) \mu_{v b}\right)\right) \\
& I_{a}^{*}=\left(\begin{array}{l}
-\gamma_{B B} \gamma_{H B} Q_{b}\left(\alpha_{h a}+\mu_{d}+\mu_{h}\right) \mu_{v a}^{2}-\gamma_{B B} \\
\left.-N_{h} \gamma_{A A} \gamma_{H A} Q_{a} \kappa+\left(\mu_{d}+\mu_{h}\right)\left(\alpha_{h a}+\mu_{d}+\mu_{h}\right) \mu_{v a}^{2}\right) \\
\mu_{v b}+\gamma_{A A} \gamma_{H A} Q_{a}\left(\alpha_{h b}+\mu_{d}+\mu_{h}\right) \mu_{v b}^{2}
\end{array}\right) \\
& /\left(\gamma_{A A} \gamma_{B B}\left(\alpha_{h a}+\mu_{d}+\mu_{h}\right)\left(\begin{array}{l}
\gamma_{H B} Q_{b} \mu_{v a}+\gamma_{H A} Q_{a} \mu_{v b} \\
\left.+\left(\mu_{d}+\mu_{h}\right) \mu_{v a}\right) \mu_{v b}
\end{array}\right)\right)
\end{aligned}
$$

And:

$$
\begin{array}{ll}
\gamma_{H A}=\omega_{a}\left(1+\lambda_{a}(\sin \eta t)\right), & \gamma_{H B}=\omega_{b}\left(1+\lambda_{b}(\sin \eta t)\right) \\
\gamma_{A A}=\beta_{v a}\left(1+\lambda_{v a}(\sin \eta t)\right), & \gamma_{B B}=\beta_{v b}\left(1+\lambda_{v b}(\sin \eta t)\right)
\end{array}
$$

The basic reproduction number for system (1) - (8), is given by:

$$
R_{0}=\frac{\gamma_{A A} \gamma_{H A} Q_{a} \mu_{v a}\left(N_{h} \gamma_{B B} K+\left(\alpha_{h b}+\mu_{d}+\mu_{h}\right) \mu_{v b}\right)}{\gamma_{B B}\left(\alpha_{h b}+\mu_{d}+\mu_{h}\right) \mu_{v a}^{2}\left(\gamma_{H B} Q_{b}+\left(\mu_{d}+\mu_{h}\right) \mu_{v b}\right)}
$$

\section{Global Stability of the Disease Free Equilibrium}

We study the global behavior of the disease free equilibrium state for the system defined by equations (1) - (8). We define new time dependent components of the death rate of the mosquitoes infected with the virus as:

$$
\left\{\begin{aligned}
\left(\mu_{v a}\right)(t)= & \omega_{a}\left(1+\lambda_{a} \sin \eta t\right) S^{*}, \\
\left(\mu_{v b}\right)(t)= & \omega_{b}\left(1+\lambda_{b} \sin \eta t\right) S^{*}, \\
\left(\mu_{d}+\mu_{h}\right)(t)= & \beta_{v a}\left(1+\lambda_{v a} \sin \eta t\right) S_{v a}^{*}, \\
& \beta_{v b}\left(1+\lambda_{v b} \sin \eta t\right) S_{v b}^{*}
\end{aligned}\right.
$$

Then Equations (1), (5) and (7) reduce to:

$$
\begin{aligned}
\frac{d S}{d t}= & \kappa N_{h}-\mu_{h}{ }^{\prime}(t) S \text { where } \mu_{h}^{\prime}(t) \\
& =\mu_{h}+\mu_{a}+\omega_{v_{a}}\left(1+\lambda_{a} \sin \eta t\right) I_{v a}+\omega_{v_{b}}\left(1+\lambda_{b} \sin \eta t\right) I_{v b}
\end{aligned}
$$




$$
\begin{aligned}
\frac{d S_{v a}}{d t} & =Q_{a}-\mu_{v a}^{\prime}(t) S_{v a} \text { where } \mu_{v a}^{\prime}(t) \\
& =\mu_{v a}+\beta_{v a}\left(1+\lambda_{v a} \sin \eta t\right) I_{a}
\end{aligned}
$$

And:

$$
\begin{aligned}
\frac{d S_{v b}}{d t}=Q_{b} & -\mu_{v b}^{\prime}(t) S_{v a} \text { where } \mu_{v a}^{\prime}(t) \\
& =\mu_{v a}+\beta_{v a}\left(1+\lambda_{v a} \sin \eta t\right) I_{a}
\end{aligned}
$$

with the rest of the equations remaining the same.

\section{Theorem 1}

When $R_{0} \leq 1$, the disease free equilibrium $J_{1}$ is globally asymptotically stable on $\Omega_{a}$.

\section{Proof}

Working now with the new set of equations to construct the Lyapunov function on $\Omega_{a}$., we get:

$$
\begin{aligned}
\psi(t) & =\left(S-S^{*} \ln S\right)+I_{a}+I_{b}+R \\
& +\left(S_{v a}-S_{v a}^{*} \ln S_{v a}\right)+I_{v a}+\left(S_{v b}-S_{v b}^{*} \ln S_{v b}\right) I_{v b}
\end{aligned}
$$

The derivative with respect to time yields:

$$
\begin{aligned}
\frac{d \psi(t)}{d t}= & \frac{d S}{d t}\left(1-\frac{S^{*}}{S}\right)+\frac{d I_{a}}{d t}+\frac{d I_{b}}{d t}+\frac{d R}{d t}+\frac{d S_{v a}}{d t}\left(1-\frac{S_{v a}^{*}}{S_{v a}}\right) \\
+\frac{d I_{v a}}{d t}+ & \frac{d S_{v b}}{d t}\left(1-\frac{S_{v b}^{*}}{S_{v b}}\right)+\frac{d I_{v b}}{d t} \\
\frac{d \psi(t)}{d t}= & \left(\begin{array}{c}
\kappa N_{h}-\omega_{a}\left(1+\lambda_{a} \sin \eta t\right) I_{v a} S-\mu_{d} S \\
\end{array}-\mu_{h} S-\omega_{b}\left(1+\lambda_{b} \sin \eta t\right) I_{v b} S\right)\left(1-\frac{S^{*}}{S}\right) \\
& +\left(\omega_{a}\left(1+\lambda_{a} \sin \eta t\right) I_{v a} S-\mu_{d} I_{a}-\mu_{h} I_{a}-\alpha_{h a} I_{a}\right) \\
& +\left(\omega_{b}\left(1+\lambda_{b} \sin \eta t\right) I_{v b} S-\mu_{d} I_{b}-\mu_{h} I_{b}-\alpha_{h b} I_{b}\right) \\
& +\left(-\mu_{d} R-\mu_{h} R-\alpha_{h a} I_{a}-\alpha_{h b} I_{b}\right) \\
& +\left(Q_{a}-\beta_{v a}\left(1+\lambda_{v a} \sin \eta t\right) S_{v a} I_{a}-\mu_{v a} S_{v a}\right)\left(1-\frac{S_{v a}^{*}}{S_{v a}}\right) \\
& +\left(\beta_{v a}\left(1+\lambda_{v a} \sin \eta t\right) S_{v a} I_{a}-\mu_{v a} I_{v a}\right) \\
& +\left(Q_{b}-\beta_{v b}\left(1+\lambda_{v b} \sin \eta t\right) S_{v b} I_{b}-\mu_{v b} S_{v b}\right)\left(1-\frac{S_{v b}^{*}}{S_{v b}}\right) \\
& +\left(\beta_{v b}\left(1+\lambda_{v b} \sin \eta t\right) S_{v b} I_{b}-\mu_{v b} I_{v b}\right)
\end{aligned}
$$

Rearranging the terms in the above expression, we get:

$$
\begin{aligned}
\frac{d \psi(t)}{d t}= & \kappa N_{h}\left(1-\frac{S^{*}}{S}\right)+Q_{a}\left(1-\frac{S_{v a}^{*}}{S_{v a}}\right)+Q_{b}\left(1-\frac{S_{v b}^{*}}{S_{v b}}\right) \\
& +I_{v a}\left(\omega_{a}\left(1+\lambda_{a} \sin \eta t\right) S^{*}-\mu_{v a}\right) \\
& +I_{v b}\left(\omega_{b}\left(1+\lambda_{b} \sin \eta t\right) S^{*}-\mu_{v b}\right)
\end{aligned}
$$

$$
\begin{aligned}
& +I_{a}\left(\beta_{v a}\left(1+\lambda_{v a} \sin \eta t\right) S_{v a}^{*}-\left(\mu_{d}+\mu_{h}\right)\right) \\
& +\left(\beta_{v b}\left(1+\lambda_{v b} \sin \eta t\right) S_{v b}^{*}-\left(\mu_{d}+\mu_{h}\right)\right) \\
& +\mu_{h} S^{*}\left(1-\frac{S}{S^{*}}\right)+\mu_{d} S^{*}\left(1-\frac{S}{S^{*}}\right) \\
& +\mu_{v a} S_{v a}^{*}\left(1-\frac{S_{v a}}{S_{v a}^{*}}\right)+\mu_{v b} S_{v b}^{*}\left(1-\frac{S_{v b}}{S_{v b}^{*}}\right)-\mu_{d} R-\mu_{h} R
\end{aligned}
$$

Using the new equilibrium states obtained from Equation (1'), (5') and (7') $S^{*}=\frac{\kappa N_{h}}{\mu_{d}+\mu_{h}}, S_{v a}^{*}=\frac{Q_{a}}{\mu_{v a}}$ and $S_{v b}^{*}=\frac{Q_{b}}{\mu_{v b}}$, expression becomes:

$$
\begin{aligned}
\frac{d \psi(t)}{d t}=\kappa & N_{h}\left(1-\frac{S^{*}}{S}\right)+Q_{a}\left(1-\frac{S_{v a}^{*}}{S_{v a}}\right)+Q_{b}\left(1-\frac{S_{v b}^{*}}{S_{v b}}\right) \\
& +\mu_{h} S^{*}\left(1-\frac{S}{S^{*}}\right)+\mu_{d} S^{*}\left(1-\frac{S}{S^{*}}\right) \\
& +\mu_{v a}(t) S_{v a}^{*}\left(1-\frac{S_{v a}}{S_{v a}^{*}}\right)+\mu_{v b}(t) S_{v b}^{*}\left(1-\frac{S_{v b}}{S_{v b}^{*}}\right) \\
& -\mu_{d} R-\mu_{h} R
\end{aligned}
$$

$$
\begin{aligned}
\frac{d \psi(t)}{d t}= & \kappa N_{h}\left(2-\frac{S^{*}}{S}-\frac{S}{S^{*}}\right)+Q_{a}\left(2-\frac{S_{v a}^{*}}{S_{v a}}-\frac{S_{v a}}{S_{v a}^{*}}\right) \\
& +Q_{b}\left(2-\frac{S_{v b}^{*}}{S_{v b}}-\frac{S_{v b}}{S_{v b}^{*}}\right)-\mu_{d} R-\mu_{h} R \\
\frac{d \psi(t)}{d t}= & -\kappa N_{h} \frac{\left(S^{*}-S\right)^{2}}{S^{*} S}-Q_{a} \frac{\left(S_{v a}^{*}-S_{v a}\right)^{2}}{S_{v a}^{*} S_{v a}} \\
& -Q_{b} \frac{\left(S_{v b}^{*}-S_{v b}\right)^{2}}{S_{v b}^{*} S_{v b}}-\mu_{d} R-\mu_{h} R
\end{aligned}
$$

We can see that all terms in (23) are always nonpositive. Using the LaSalle's extension to Lyapunov's theorem, we have $\frac{d \psi(t)}{d t} \leq 0$ and so the function $\frac{d \psi(t)}{d t}$ is seen to be negative definite. The limit set of each solution is contained in the largest invariant set for which $S=S^{*}, S_{v a}=S_{v a}^{*}, S_{v b}=S_{v b}^{*}$ and $R^{n}=0$ which is the singleton $\left\{J_{1}\right\}$. Then, the LaSalle's invariant principle implies that the disease free equilibrium $J_{1}$ is globally asymptotically stable on $\Omega_{a}$.

Next, we consider the global property of the endemic equilibrium of (1)-(8).

\section{Theorem 2}

If $R_{0}>1$, the endemic equilibrium state $J_{2}\left(S^{*}, I_{a}^{*}, I_{b}^{*}, R^{*}, S_{v a}^{*}, I_{v a}^{*}, S_{v b}^{*}, I_{v b}^{*}\right) \in \Omega_{a}$ exists and is globally asymptotically stable on $\Omega_{a}$. To see this, we now 
redefine the time dependent components of death rates, Equation (20), as:

$$
\left\{\begin{array}{l}
\mu_{v a}(t)=\left(\omega_{a}\left(1+\lambda_{a} \sin \eta t\right) N_{h}\right) \\
\mu_{v b}(t)=\left(\omega_{b}\left(1+\lambda_{a} \sin \eta t\right) N_{h}\right) \\
\left(\mu_{d}+\mu_{h}+\alpha_{h a}\right)(t)=Q \beta_{v a}\left(1+\lambda_{v a} \sin \eta t\right) S_{v a}^{*} \\
\left(\mu_{d}+\mu_{h}+\alpha_{h b}\right)(t)=Q \beta_{v b}\left(1+\lambda_{v b} \sin \eta t\right) S_{v b}^{*}
\end{array}\right.
$$

\section{Proof}

The Lyapunov function is in the form:

$$
\begin{aligned}
& \rho(t)=\left(S-S^{*} I n S\right)+I_{a}+I_{b} \\
& +\left(\frac{\mu_{d}+\mu_{h}+\alpha_{h a}+\alpha_{h b}}{A_{1} S_{v a}^{*}+A_{2} S_{v b}^{*}}\right)\left(S_{v a}-S_{v a}^{*} \operatorname{In} S_{v a}\right) \\
& +\left(\frac{\mu_{d}+\mu_{h}+\alpha_{h a}+\alpha_{h b}}{A_{1} S_{v a}^{*}+A_{2} S_{v b}^{*}}\right) I_{v a} \\
& +\left(\frac{\mu_{d}+\mu_{h}+\alpha_{h a}+\alpha_{h b}}{A_{1} S_{v a}^{*}+A_{2} S_{v b}^{*}}\right)\left(S_{v b}-S_{v b}^{*} \operatorname{In} S_{v b}\right) \\
& +\left(\frac{\mu_{d}+\mu_{h}+\alpha_{h a}+\alpha_{h b}}{A_{1} S_{v a}^{*}+A_{2} S_{v b}^{*}}\right) I_{v b}
\end{aligned}
$$

With:

$$
\begin{aligned}
& \mathrm{A}_{1}(\mathrm{t})=\omega_{\mathrm{a}}\left(1+\lambda_{\mathrm{a}} \sin \eta \mathrm{t}\right) \\
& \mathrm{A}_{2}(\mathrm{t})=\omega_{\mathrm{b}}\left(1+\lambda_{\mathrm{b}} \sin \eta \mathrm{t}\right)
\end{aligned}
$$

Its derivative along the trajectories of (1)-(8):

$$
\begin{aligned}
& \frac{d \rho(t)}{d t}=\frac{d S}{d t}\left(1-\frac{S^{*}}{S}\right)+\frac{d I_{a}}{d t}+\frac{d I_{b}}{d t} \\
& +\left(\frac{\mu_{d}+\mu_{h}+\alpha_{h a}+\alpha_{h b}}{A_{1} S_{v a}^{*}+A_{2} S_{v b}^{*}}\right) \\
& \frac{d S_{v a}}{d t}\left(1-\frac{S_{v a}^{*}}{S_{v a}}\right)+\left(\frac{\mu_{d}+\mu_{h}+\alpha_{h a}+\alpha_{h b}}{A_{1} S_{v a}^{*}+A_{2} S_{v b}^{*}}\right) \frac{d I_{v a}}{d t} \\
& +\left(\frac{\mu_{d}+\mu_{h}+\alpha_{h a}+\alpha_{h b}}{A_{1} S_{v a}^{*}+A_{2} S_{v b}^{*}}\right) \frac{d S_{v b}}{d t}\left(1-\frac{S_{v b}^{*}}{S_{v b}}\right) \\
& +\left(\frac{\mu_{d}+\mu_{h}+\alpha_{h a}+\alpha_{h b}}{A_{1} S_{v a}^{*}+A_{2} S_{v b}^{*}}\right) \frac{d I_{v b}}{d t} \\
& \frac{d \rho(t)}{d t}=\left(\begin{array}{l}
\kappa N_{h}-\omega_{a}\left(1+\lambda_{a} \sin \eta t\right) I_{v a} S \\
-\mu_{d} S-\mu_{h} S-\omega_{b}\left(1+\lambda_{b} \sin \eta t\right) I_{v b} S
\end{array}\right)\left(1-\frac{S^{*}}{S}\right) \\
& +\left(\omega_{a}\left(1+\lambda_{a} \sin \eta t\right) I_{v a} S-\mu_{d} I_{a}-\mu_{h} I_{a}-\alpha_{h a} I_{a}\right) \\
& +\left(\omega_{b}\left(1+\lambda_{b} \sin \eta t\right) I_{v b} S-\mu_{d} I_{b}-\mu_{h} I_{b}-\alpha_{h b} I_{b}\right) \\
& +\left(\frac{\mu_{d}+\mu_{h}+\alpha_{h a}+\alpha_{h b}}{A_{1} S_{v a}^{*}+A_{2} S_{v b}^{*}}\right)\left(\begin{array}{l}
Q_{a}-\beta_{v a}\left(1+\lambda_{v a} \sin \eta t\right) \\
S_{v a} I_{a}-\mu_{v a} S_{v a}
\end{array}\right)\left(1-\frac{S_{v a}^{*}}{S_{v a}}\right)
\end{aligned}
$$

$$
\begin{aligned}
& +\left(\frac{\mu_{d}+\mu_{h}+\alpha_{h a}+\alpha_{h b}}{A_{1} S_{v a}^{*}+A_{2} S_{v b}^{*}}\right)\left(\beta_{v a}\left(1+\lambda_{v a} \sin \eta t\right) S_{v a} I_{a}-\mu_{v a} I_{v a}\right) \\
& +\left(\frac{\mu_{d}+\mu_{h}+\alpha_{h a}+\alpha_{h b}}{A_{1} S_{v a}^{*}+A_{2} S_{v b}^{*}}\right)\left(\begin{array}{l}
Q_{b}-\beta_{v b}\left(1+\lambda_{v b} \sin \eta t\right) \\
S_{v b} I_{b}-\mu_{v b} S_{v b}
\end{array}\right)\left(1-\frac{S_{v b}^{*}}{S_{v b}}\right) \\
& +\left(\frac{\mu_{d}+\mu_{h}+\alpha_{h a}+\alpha_{h b}}{A_{1} S_{v a}^{*}+A_{2} S_{v b}^{*}}\right)\left(\begin{array}{l}
\beta_{v b}\left(1+\lambda_{v b} \sin \eta t\right) \\
S_{v b} I_{b}-\mu_{v b} I_{v b}
\end{array}\right)
\end{aligned}
$$

Since we assume that total number of populations are constants, so we have $\kappa N_{h}=N_{T}\left(\mu_{d}+\mu_{h}\right), Q_{a}=$ $N_{v a} \mu_{v a}=\mu_{v a}\left(S_{v a}+I_{v a}\right)$ and $Q_{b}=N_{v b} \mu_{v b}=\mu_{v b}\left(S_{v b}+I_{v b}\right)$. Then above equation become:

$$
\begin{aligned}
& \frac{d \rho(t)}{d t}=\left(\mu_{d}+\mu_{h}\right) N_{T}\left(1-\frac{S^{*}}{S}\right) \\
& +Q \mu_{v a} N_{v a}\left(1-\frac{S_{v a}^{*}}{S_{v a}}\right)+Q \mu_{v b} N_{v b}\left(1-\frac{S_{v b}^{*}}{S_{v b}}\right) \\
& \left.+I_{v a}\left(\omega_{a}\left(1+\lambda_{a} \sin \eta t\right) N_{h}\right)-Q \mu_{v a} \frac{S_{v a}^{*}}{S_{v a}}\right) \\
& \left.+I_{v b}\left(\omega_{b}\left(1+\lambda_{b} \sin \eta t\right) N_{h}\right)-Q \mu_{v b} \frac{S_{v b}^{*}}{S_{v b}}\right) \\
& +I_{a}\left(Q \beta_{v a}\left(1+\lambda_{v a} \sin \eta t\right) S_{v a}^{*}-\left(\mu_{d}+\mu_{h}+\alpha_{h a}\right)\right) \\
& +I_{b}\left(Q \beta_{v b}\left(1+\lambda_{v b} \sin \eta t\right) S_{v b}^{*}-\left(\mu_{d}+\mu_{h}+\alpha_{h b}\right)\right)
\end{aligned}
$$

$$
\text { when } Q=\left(\frac{\mu_{d}+\mu_{h}+\alpha_{h a}+\alpha_{h b}}{A_{1} S_{v a}^{*}+A_{2} S_{v b}^{*}}\right) \text {. }
$$

Substituting conditions (24) into (28), we have:

$$
\begin{aligned}
& \frac{d \rho(t)}{d t}=\left(\mu_{d}+\mu_{h}\right) N_{T}\left(1-\frac{S^{*}}{S}\right) \\
& +Q \mu_{v a} S_{v a}\left(1-\frac{S_{v a}^{*}}{S_{v a}}\right)+Q_{1}\left(1-\frac{S_{v a}^{*}}{S_{v a}}-\frac{S_{v a}}{S_{v a}^{*}}\right) \\
& +Q S_{v b} \mu_{v b}\left(1-\frac{S_{v b}^{*}}{S_{v b}}\right)+Q_{2}\left(1-\frac{S_{v b}^{*}}{S_{v b}}-\frac{S_{v b}}{S_{v b}^{*}}\right) \\
& +I_{v a}\left(\left(\omega_{a}\left(1+\lambda_{a} \sin \eta t\right) N_{h}\right)-\mu_{v a}\right) \\
& +I_{v b}\left(\left(\omega_{b}\left(1+\lambda_{b} \sin \eta t\right) N_{h}\right)-\mu_{v b}\right) \\
& +I_{a} Q \beta_{v a}\left(1+\lambda_{v a} \sin \eta t\right) S_{v a}^{*}-\left(\mu_{d}+\mu_{h}+\alpha_{h a}\right) I_{a} \\
& +I_{b} Q \beta_{v b}\left(1+\lambda_{v b} \sin \eta t\right) S_{v b}^{*}-\left(\mu_{d}+\mu_{h}+\alpha_{h b}\right) I_{b}
\end{aligned}
$$

In $\Omega_{a}$, we have $Q_{1}=I_{v a} Q \mu_{v a}$ and $Q_{2}=I_{v b} Q \mu_{v b}$. The above equation (29) becomes:

$$
\begin{aligned}
& \frac{d \rho(t)}{d t}=\left(\mu_{d}+\mu_{h}\right) N_{T}\left(1-\frac{S^{*}}{S}\right) \\
& +Q \mu_{v a}(t) S_{v a}\left(1-\frac{S_{v a}^{*}}{S_{v a}}\right)+Q_{1}\left(1-\frac{S_{v a}^{*}}{S_{v a}}-\frac{S_{v a}}{S_{v a}^{*}}\right) \\
& +Q S_{v b} \mu_{v b}(t)\left(1-\frac{S_{v b}^{*}}{S_{v b}}\right)+Q_{2}\left(1-\frac{S_{v b}^{*}}{S_{v b}}-\frac{S_{v b}}{S_{v b}^{*}}\right)
\end{aligned}
$$




$$
\begin{aligned}
& +I_{v a}\left(\left(\omega_{a}\left(1+\lambda_{a} \sin \eta t\right) N_{h}\right)-\mu_{v a}(t)\right) \\
& +I_{v b}\left(\left(\omega_{b}\left(1+\lambda_{b} \sin \eta t\right) N_{h}\right)-\mu_{v b}(t)\right) \\
& +I_{a} Q \beta_{v a}\left(1+\lambda_{v a} \sin \eta t\right) S_{v a}^{*}-\left(\mu_{d}+\mu_{h}+\alpha_{h a}\right)(t) I_{a} \\
& +I_{b} Q \beta_{v b}\left(1+\lambda_{v b} \sin \eta t\right) S_{v b}^{*}-\left(\mu_{d}+\mu_{h}+\alpha_{h b}\right)(t) I_{b} \\
& \frac{d \rho(t)}{d t}=-\left(\mu_{d}+\mu_{h}\right) N_{T} \frac{\left(S-S^{*}\right)^{2}}{S S^{*}} \\
& -Q \mu_{v a}(t) S_{v a} \frac{\left(S_{v a}-S_{v a}^{*}\right)^{2}}{S_{v a} S_{v a}^{*}}-Q_{1} \frac{\left(S_{v a}-S_{v a}^{*}\right)^{2}}{S_{v a} S_{v a}^{*}} \\
& -Q S_{v b} \mu_{v b}(t) \frac{\left(S_{v b}-S_{v b}^{*}\right)^{2}}{S_{v b} S_{v b}^{*}}-Q_{2} \frac{\left(S_{v b}-S_{v b}^{*}\right)^{2}}{S_{v b} S_{v b}^{*}} \\
& \frac{d \rho(t)}{d t}=\left(\mu_{d}+\mu_{h}\right) N_{T}\left(2-\frac{S^{*}}{S}-\frac{S}{S^{*}}\right) \\
& +Q \mu_{v a}(t) S_{v a}\left(2-\frac{S_{v a}^{*}}{S_{v a}}-\frac{S_{v a}}{S_{v a}^{*}}\right)+Q_{1}\left(2-\frac{S_{v a}^{*}}{S_{v a}}-\frac{S_{v a}}{S_{v a}^{*}}\right) \\
& +Q S_{v b} \mu_{v b}(t)\left(2-\frac{S_{v b}^{*}}{S_{v b}}-\frac{S_{v b}}{S_{v b}^{*}}\right)+Q_{2}\left(2-\frac{S_{v b}^{*}}{S_{v b}}-\frac{S_{v b}}{S_{v b}^{*}}\right)
\end{aligned}
$$

We use the LaSalle's invariant principle to show that $\frac{d \rho(t)}{d t} \leq 0$ for all $\left(S^{*}, I_{a}^{*}, I_{b}^{*}, R^{*}, S_{v a}^{*}, I_{v a}^{*}, S_{v b}^{*}, I_{v b}^{*}\right) \in \Omega_{a}$ and the strict equality $\frac{d \rho(t)}{d t}=0$ holds only for $S=S^{*}, I_{a}=I_{a}^{*}, I_{b}=I_{b}^{*}, R=R^{*}, S_{v a}=S_{v a}^{*}, I_{v a}=I_{v a}^{*}, S_{v b}=S_{v b}^{*} \quad$ and $I_{v b}=I_{v b}^{*}$. Then, the equilibrium state $J_{2}$ is the only invariant set of solutions of the equations (1)-(8) contained entirely in $\left\{\begin{array}{l}\left(S^{*}, I_{a}^{*}, I_{b}^{*}, R^{*}, S_{v a}^{*}, I_{v a}^{*}, S_{v b}^{*}, I_{v b}^{*}\right), S=S^{*}, I_{a}=I_{a}^{*}, I_{b}=I_{b}^{*}, R=R^{*}, \\ S_{v a}=S_{v a}^{*}, I_{v a}=I_{v a}^{*}, S_{v b}=S_{v b}^{*} \text { and } I_{v b}=I_{v b}^{*}\end{array}\right\}$ and hence the asymptotic stability theorem, the positive endemic equilibrium state $J_{2}$ is global asymptotic stability in $\Omega_{a}$.

\section{Discussion and Conclusion}

Let:

$$
R_{0}=\frac{\gamma_{A A} \gamma_{H A} Q_{a} \mu_{v a}\left(N_{h} \gamma_{B B} \kappa+\left(\alpha_{h b}+\mu_{d}+\mu_{h}\right) \mu_{v b}\right)}{\gamma_{B B}\left(\alpha_{h a}+\mu_{d}+\mu_{h}\right) \mu_{v b}^{2}\left(\gamma_{H B} Q_{b}+\left(\mu_{d}+\mu_{h}\right) \mu_{v b}\right)}
$$

be the threshold parameters. Then, we define $R_{0}=\sqrt{R_{0}}$ as the basic reproductive number of disease. Also, it represents the average number of secondary cases produced from susceptible population. We consider human and vector (A. aegypti and Aedes albopictus) populations. It depends on the transmission rate of dengue virus.
In this paper, we have studied a mathematical model of dengue disease in which the virus is being transmitted by two different species of the Aedes mosquitoes by looking at the global stability of our model. The global stability of transmission of dengue disease in human and vector (Aedes aegypti and A. albopictus) was determined been by using Lyapunov functions. If $R_{0} \leq 1$, then to the disease-free equilibrium state is globally asymptotically stable. In the feasible region and the disease dies out of population. If $R_{0}>1$, then there is the unique endemic equilibrium state which is globally asymptotically stable in the interior of feasible region and the disease is present. If the disease is present in the population, then it will persist. Nothing in the model depends directly on the dengue virus directly except for the use of a sinusoidal variation of the transmission rates which were inferred by the behavior seen in Figure 1. Since the ZIKA epidemic has only been around for less than a year, there has not been enough time to observe what the seasonal dependence is. When that information becomes available, the present work can be modified so that it can be applied to possible ZIKA pandemic which might arise in the near future.

\section{Acknowledgment}

This study is supported by King Mongkut's Institute of Technology Ladkrabang and National Research Council of Thailand. IMT acknowledge the financial support provided by King Mongkut's University of Technology, Thonburi through the KMUTT 55th Anniversary Commemorative Fund.

\section{Author's Contributions}

Assoc. Prof. Dr. Puntani Pongsumpun: was the advisor of Rattiya Sugchasit who did the work for her $\mathrm{Ph}$.D. degree. She guided the student on a day to day basis and wrote the first draft of the paper with Ms. Rattiya Sungchasit.

Dr. Rattiya Sungchasit: did the work for her Ph.D. which has received. She did the analysis the model and carried out the computer calculations. She is the one who suggested using the Lyapanov function method to establish the global stability of the model.

Prof. Dr. I-Ming Tang: was the person who suggested the problem, the spread of the ZIKA virus and the mathematical model to describe the transmission of this disease and its connection the spread of the dengue disease. He is responsible for the incorporation of the two species of the Aedes mosquitoes. The final version of the paper was written by him. 


\section{Conflict of Interests}

The authors declare that there is no conflict of interest regarding this research.

\section{References}

Bardina, S.V., P. Bunduc, S. Tripathi, J. Duehr and J.J. Frere et al., 2017. Enhancement of Zika virus pathogenesis by preexisting antiflavivirus immunity. Science, 356: 175-180.

DOI: $10.1126 /$ science.aal4365

Benedict, M.Q., R.S. Levine, W.A. Hawley and P. Lounibos, 2007. Spread of the Tiger: Global Risk of Invasion by mosquito Aedes albopictus. Vector Borne Zoonotic Dis., 7: 76-85.

Beretta, E. and V. Capasso, 1986. On the general structure of epidemic systems: Global asymptotic stability. Comp. Maths. Appls., 12: 677-694. DOI: 10.1016/0898-1221(86)90054-4

Chadee, D.D., 2004. Key premises, a guide to Aedes aegypti (Diptera:Culicidae) surveillance and control. Bull. Entomol. Res., 94: 201-207.

DOI: 10.1079/BER2004297

Costanzo, K.S., B. Kesavaraju and S.A. Juliano, 2005. Condition-specific competition in container mosquitoes: The role of non-peting life-history stages. Ecology, 86: 3289-3295.

D'Ortenzio, E., S. Matheron, X. de Lamballerie, B. Hubert and G. Piorkowski et al., 2016. Evidence of sexual transmission of Zika virus. N. Engl. J. Med., 374: 2195-2198. DOI: 10.1056/NEJMc1604449

Davidson, A., S. Slavinski, K. Komoto, J. Rakeman and D. Weiss, 2016. Suspected female-to-male sexual transmission of Zika Virus. New York.

Diekmann, O. and J.A.P. Heesterbeek, 2000. Mathematical Epidemiology of Infectious Disease: Model Building, Analysis and Interpretation. 1st Edn., John Wiley, ISBN: 10: 0471492418, pp: 320.

Division of Epidemiology, 2002-2011. Annual epidemiological survillance report. Ministry of Public Health: Ministry of Public Health Thailand.

Fauci, A.S. and D.M. Morens, 2016. Zika Virus in the Americas- yet another Arbovirus threat. N. Engl. J. Med., 347: 601-604. DOI: 10.1056/NEJMp1600297

Fisman, D.N., 2007. Seasonality of infectious diseases. Annu. Rev. Public. Health, 28: 127-143.

Greenfell, B.T., B.M. Bolker and A. Kleczkowski, 1995. Seasonslity and Extinctin in Chaotic Metapopulations. Proc. Biol. Sci., 259: 97-103. DOI: $10.1098 / \mathrm{rspb} .1995 .0015$

Gubler, D.J., 1998. Dengue and dengue hemorrhagic fever. Clin. Microbiol. Rev., 11: 480-496.

Hawley, W.A., 1988. The biology of Aedes albopictus. J. Am. Mosquito Con. Assoc., 1: 11-39.
Juliano, S.A., G.F. O'Meara, J.R. Morril and M.M. Cutwa, 2002. Desiccation and thermal tolerance of eggs and the coexistence of competing mosquitoes. Oecologia, 130: 458-469.

Keeling, M.J. and P. Rohani, 2008. Modeling Infectious Diseases in Humans and Animals. Princeton University Press, ISBN: 10: 0691116172, pp: 366.

Koenraadt, C. J M., J. Aldstadt, U. Kijchalao, A. Kengluecha and J.W. Jones et al., 2007. Spatial and Temporal Patterns in the Recovery of Aedes aegypti (Diptera: Culicidae) Populations after Insecticide Treatment. J. Med. Entomol., 44: 65-71. DOI: $10.1603 / 0022-$ 2585(2007)44[65:SATPIT]2.0.CO;2

LaSalle, J. and S. Lefschetz, 1961. Stability by Liapunov's Direct Method. Academic Press, New York, pp: 134.

Li, M.K. and J. Muldowney, 1995. Global stability for the SEIR model in epicemiology. Math. Bio. Sci., 125: 155-164. DOI: 10.1016/0025-5564(95)92756-5

Littaua, R., I Kurane and F.A. Ennis, 1990. Human IgG $\mathrm{Fc}$ receptor II mediates antibody dependent enhancement of dengue virus infection. J. Immunol., 144: 3183-3186.

McCracken, M.K., G.D. Gromowski, H.L. Friberg, X. Lin and P. Abbink et al., 2017. Impact of prior flavivirus immunity on Zika virus infection in rhesus macaques, PLoS Pathog, 13: 64-87. DOI: 10.1371/journal.ppat.1006487

Mliakar, J., 2016. Zika virus associated with microcephaly. Engl. J. Med., 374: 951-958. DOI: 10.1056/NEJMoa1600651

Mori, A. and Y .Wada, 1978. The season abundance of aedes albopictus in nagasaki. Tropical Medicine.

Musso, D., C. Roche, E. Robin, T. Nhan and A. Teissier et al., 2015. Potential sexual transmission of Zika virus. Emerg. Inf. Dis., 21: 359-361. DOI: $10.3201 /$ eid2102.141363

New York Times, 2016. https://www.nytimes.com/2016/02/03/opinion/fighti ng-the-zika-virus.html

Peterson, L.R., D.J. Jamise, A.M. Powers, M.A. Honein and Z. Virus, 2016. NEJM 374, 1552.

Pierson, T.C. and B.S. Graham, 2016. Zika Virus: Immunity and vaccine development. Cell, 167: 625-631. DOI: 10.1016/j.cell.2016.09.020

Priyamvada, L., 2016. Human antibody responses after dengue infection are highly cross reactive to Zika Virus. PNAS 113: 7852-7857. DOI: $10.1073 /$ pnas.1607931113

Rasmussen, S.A., D.J. Jamieson, M.A. Honein and L.R. Petersen, 2016. Zika virus and birth defectsreviewing the evidence for causality. N. Engl. J. Med., 374: 1981-1987.

DOI: 10.1056/NEJMsr1604338 
Guga-Sapir, D. and B. Schimmer, 2005. Dengue fever, new paradigms for a changing epidemiology. Emerg. Themes Epidemiol. 2: 1-9.

DOI: $10.1186 / 1742-7622-2-1$

Science News, 2016.

https://www.sciencenews.org/article/unknownsabout-zika-virus-continuefrustrate mode $=$ magazine $\&$ context $=3070$

Serpa, L.L., G.R.A.M. Margues, A.P. de Lima, J.C. Voltolina and M. d Brdo et al., 2013. Study of the distribution and abundance of the eggs of Aedes aegypti and Aedes albopictus according to the habitat and meteorological variables, municipality of Sao Sebastiao. Sao Paulo State Brazil. Prasite Vec. 6: 121-321. DOI: 10.1186/1756-3305-6-321

Simpson, D.J.H., 1964. Zika virus infection in man. Trans. R. Soc. Trop. Med. Hyg., 58: 335-338.

DOI: $10.1016 / 0035-9203(64) 90200-7$
Sungchasit, R., P. Pongsumpun and I.M. Tang, 2013. Transmission model of dengue Virus aedes aegypti and aedes albopictus. Far East J. Mathema. Sci., 83: 85.

Turmel, J.M., P. Abgueguen, B. Hubert, Y.M. Vandamme and M. Maquart et al., 2016. Late sexual transmission of zika virus related to persistence in the semen. Lancet, 387: 2501-2506. DOI: $10.1016 / \mathrm{S} 0140-6736(16) 30775-9$

Vaughn, D.W., S. Green, S. Kalayanarooj, B.L. Innis and S. Nimmannitya et al., 2000. Dengue viremia titer, antibody response pattern and virus serotype correlate with disease severity. J. Infect. Dis., 181: 2-9. DOI: $10.1086 / 315215$

WHO, 2016. WHO director-general. R. Margaret Chan:\#ZikaVirus. 